\title{
Effect of geometric base roughness on size segregation
}

\author{
L. Jing ${ }^{1}$, C. Y. Kwok ${ }^{1, \star}$, Y. F. Leung ${ }^{2}$, and Y. D. Sobral ${ }^{3}$ \\ ${ }^{1}$ Department of Civil Engineering, The University of Hong Kong, Pokfulam Road, Hong Kong \\ ${ }^{2}$ Department of Civil and Environmental Engineering, The Hong Kong Polytechnic University, Hong Kong \\ ${ }^{3}$ Departamento de Matemática, Universidade de Brasília, Campus Universitário Darcy Ribeiro, 70910-900 Brasília, DF, Brazil
}

\begin{abstract}
The geometric roughness at boundaries has a profound impact on the dynamics of granular flows. For a bumpy base made of fixed particles, two major factors have been separately studied in the literature, namely, the size and spatial distribution of base particles. A recent work (Jing et al. 2016) has proposed a roughness indicator $R_{a}$, which combines both factors for any arbitrary bumpy base comprising equally-sized spheres. It is shown in mono-disperse flows that as $R_{a}$ increases, a transition occurs from slip $\left(R_{a}<0.51\right)$ to non-slip $\left(R_{a}>0.62\right)$ conditions. This work focuses on such a phase transition in bi-disperse flows, in which $R_{a}$ can be a function of time. As size segregation takes place, large particles migrate away from the bottom, leading to a variation of size ratio between flow- and base-particles. As a result, base roughness $R_{a}$ evolves with the progress of segregation. Consistent with the slip/non-slip transition in mono-disperse flows, basal sliding arises at low values of $R_{a}$ and the development of segregation might be affected; when $R_{a}$ increases to a certain level ( $R_{a}>0.62$ ), non-slip condition is respected. This work extends the validity of $R_{a}$ to bi-disperse flows, which can be used to understand the geometric boundary effect during segregation.
\end{abstract}

\section{Introduction}

Base roughness plays an important role in the flow of granular materials. For instance, the minimum thickness necessary to sustain a steady flow down incline is a function of base roughness [1]; the fingering instability developed at the front of granular avalanches is due to the change of basal resistance upon segregation [2-4]; an inadequate base roughness may be responsible for the unsteady flow regime and crystallisation in chute flows [5-7]; in smallscale experimental debris flows, base roughness is the key to produce a realistic deposition with levee formation [8].

To achieve sufficiently rough (i.e. non-slip) base conditions, geometric roughness is usually provided by gluing particles at the boundary surface. By contrast, a flat surface with only mechanical friction usually leads to a shear layer near the base, above which plug flows may occur with poorly-developed shear $[5,9,10]$. In discrete element modelling (DEM), one can construct a rough base by fixing a layer of particles with a regular/random packing [5, 9, 11-21]. For such a bumpy base, both the size and spatial distribution of the fixed particles are influential factors of the geometric roughness. To better understand the basal effect on flow dynamics, it is favorable to characterise geometric rougness in terms of these two factors. A recent work by Jing et al. [20] proposes an alternative indicator of base roughness, $R_{a}$. It is shown that in mono-disperse flows $R_{a}$ well describes the geometric roughness of any arbitrary base composing equally-sized

\footnotetext{
^e-mail: fiona.kwok@hku.hk
}

spheres; as $R_{a}$ increases, a phase transition occurs from slip $\left(R_{a}<0.51\right)$ to non-slip $\left(R_{a}>0.62\right)$ regimes, with an intermediate regime of $R_{a} \in[0.51,0.62]$.

In this paper, we adopt $R_{a}$ to study the basal effects in both mono- and bi-disperse flows. In bi-disperse flows, one issue of interest is that size segregation leads to a variation of base roughness, thus affecting flow kinetics $[19,22]$. When larger particles are initially in contact with the base (which is made of small particles), the relative roughness is low. The occurrence of size segregation and the subsequent upward migration of large particles change the relative roughness. As a result, the boundary value of velocity evolves with the progress of segregation.

The rest of this paper is organised as follows: In Sect. 2 we briefly introduce the simulation set-up, the strategy of base generation, and the characterisation of base roughness. Section 3 presents the effect of base roughness in both mono- and bi-disperse flows, with emphasis on the variation of geometric roughness during segregation and the consequence of such a variation. In Sect. 4 we conclude our findings.

\section{Characterisation of base roughness}

\subsection{Case set-up}

In our DEM simulations, periodic boundaries are imposed to both the flow $(x)$ and vorticity $(z)$ directions, and the top surface is free of constraint [Fig. 1(a)]. The base is roughened by randomly-packed particles [Fig. 1(b)]. At the initial state, a layer of small particles (diameter $d_{s}$ ) is placed 


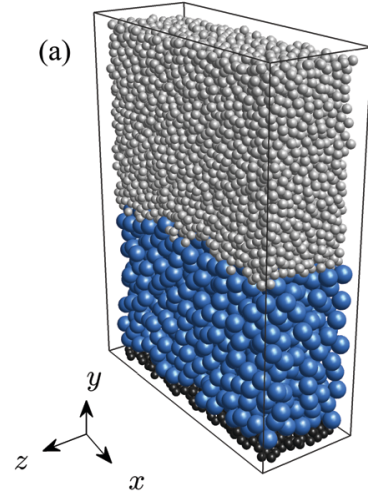

(b)

(c)

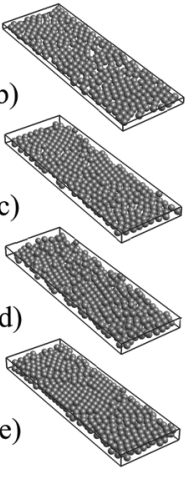

Figure 1. Simulation set-up. (a) A bi-disperse flow with periodic boundaries. (b-e) Bases constructed with $H_{b} / d_{b}=$ $1.0,1.6,1.8,2.0$, respectively.

on top of a bed of large particles (diameter $d_{l}=S_{r} d_{s}$ ), in which $S_{r}$ denotes the size ratio of the binary mixture. To initiate the flow, gravity is tilted in the $x y$ plane to a desired inclination $\theta$. The flow thickness $H$ is a function of time $t$ after the flow is initiated. Unless otherwise specified, we consistently use $d_{s}=0.005 \mathrm{~m}, H=40 d_{s}$ at $t=0$, and $\theta=25^{\circ} ; S_{r}=1$ and 2 are specified for mono- and bi-disperse flows, respectively; particle properties include density $\rho=2650 \mathrm{~kg} / \mathrm{m}^{3}$, Young's Modulus $E=5 \times 10^{6}$ $\mathrm{Pa}$ and Poisson's ratio $v=0.35$. The Hertz contact model is used, in which the coefficients of restitution and friction are $e=0.5$ and $\mu=0.5$, respectively. The two contact properties are not varied since we focus on geometric roughness in the present work. The effect of these parameters are discussed in [21].

\subsection{Base construction}

In the construction of bumpy bases, a variety of particle sizes and packings are adopted. The size ratio between flow particles (diameter $d$ ) and base particles $\left(d_{b}\right)$ is denoted by $\Phi=d / d_{b}$, in which $d$ is the average diameter of flow particles contacting the base. The (mean) spacing measured over a layer is noted as $\varepsilon_{k} d_{b}$, where $\varepsilon$ is spacing and $k=1,2,3$ represents the three sides [Fig. 2(a)]. A base can comprise multiple layers with thickness $H_{b}$ [Fig. 1(b)-1(e)]. Let $y=0$ be the top of a base, we firstly place a wall at $y=-H_{b}$, then pour base particles onto the wall, and finally trim particles beyond $y=0$. By varying $H_{b} / d_{b}$, different distributions of base particles are produced. As reported in [20], $H_{b} / d_{b}=1.8$ generally yields the roughest base for a given $\Phi$ in mono-disperse flows.

\subsection{Base roughness}

Here we present briefly the characterisation of geometric roughness. Consult Jing et al. 2016 [20] for more details.

Given a bumpy base made of particles, such as those in Figs. 1(b)-1(e), we assume that within each layer particles are centred at one common plane. We can discretise
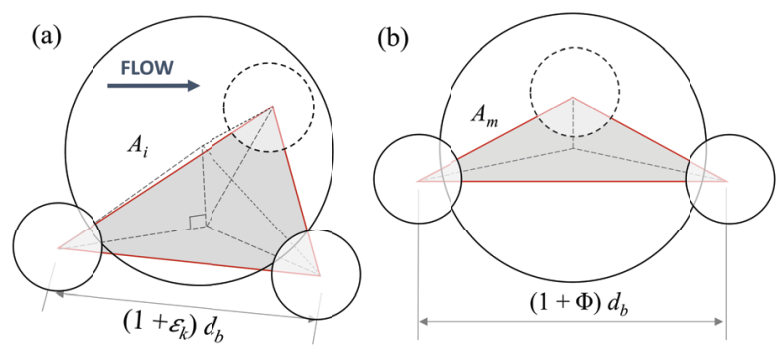

Figure 2. Definition of base roughness. The larger ball represent a flowing particle and smaller balls the fixed base particles. (a) An arbitrary void with area $A_{i}$. (b) An equilateral void with area $A_{m}$ (the most stable with respect to flow).

this plane into an assembly of triangles using the Delaunay triangulation scheme [23]. The $i$ th triangle represents a local void among three base particles (whose centroids are the vertices of the triangle), with area $A_{i}$ [Fig. 2(a)]. We interpret roughness as the resistance to motion when a particle flows over the local void. The maximum roughness is achieved when the flowing particle is coplanar with the three base particles, in which case we denote the void area as $A_{m}$ [Fig. 2(b)]. For a layer, we define its (mean) roughness as

$$
R_{a}=\frac{1}{N} \sum_{i=1}^{N} \frac{A_{i}}{A_{m}}
$$

where $R_{a}$ stands for the roughness defined by area ratio, and $N$ is the number of triangular voids. This definition combines two key steps shown in [20].

As seen in Fig. 2, $A_{i}$ can be expressed in terms of $\varepsilon_{k}$

$$
A_{i}=d_{b}^{2} \sqrt{\frac{\sum_{k}\left(1+\varepsilon_{k}\right)}{2} \prod_{k}\left[\frac{\sum_{k}\left(1+\varepsilon_{k}\right)}{2}-\left(1+\varepsilon_{k}\right)\right]}
$$

and $A_{m}=\frac{\sqrt{3}}{4}(1+\Phi)^{2} d_{b}^{2}$. Note that when $A_{i}$ exceeds a critical value, the layer underneath void $i$ also contributes to the base roughness. In this case, $A_{i} / A_{m}$ is truncated such that $R_{a} \leqslant 1$, and a non-zero weight is assigned to the underneath layer. This process is referred to as multi-layer composition and is elaborated in [20]. Clearly, $R_{a}$ is a function of spacing $\varepsilon_{k}$ and size ratio $\Phi$, with limits of zero and unity; for each layer, a wider spacing or a larger size of base particles tends to enhance the geometric roughness.

\section{Effect of base roughness}

\subsection{Mono-disperse flow}

Both the size and packing of base particles are influential to the flow kinetics [20]. As seen in Fig. 3, for the same packing $\left(H_{b} / d_{b}=1.0\right)$ but different size ratios $(\Phi=0.5,1.0,2.0)$, the velocity profiles show a significant difference. Larger base particles $(\Phi=0.5)$ provide larger voids to trap the flowing particles near the base [Fig. 3(b)], while smaller base particles $(\Phi=1.0,2.0)$ produce sliding at the base. Notably, the velocity profiles are mainly 

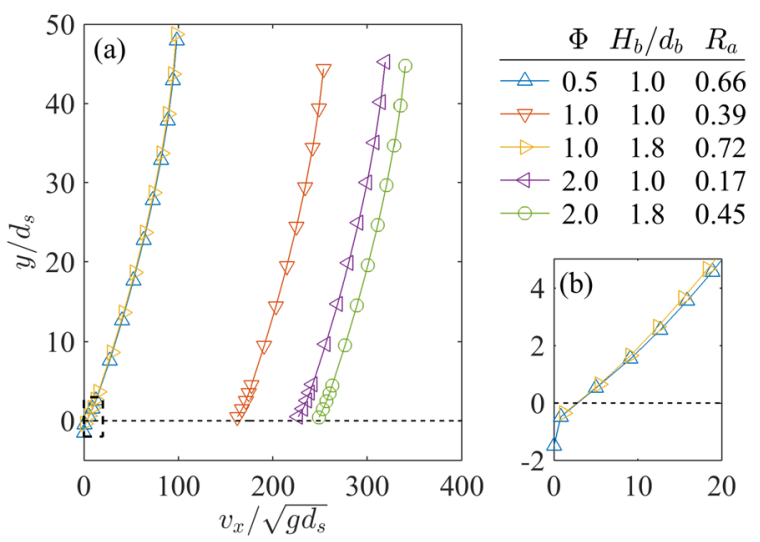

Figure 3. Basal effect on mono-disperse flows $\left(\theta=25^{\circ}\right)$. (a) Velocity profiles at steady state. (b) Details near the base surface, i.e. the region with dashed borders in (a).

shifted horizontally, but their shapes remain similar. For size ratio $\Phi=1.0, H_{b} / d_{b}=1.8$ ensures non-slip condition to the flow because more bumps are generated [Fig. 1(d)]. However, when base particles are half the size of the flowing particles, i.e. $\Phi=2.0$, the sliding can not be avoided by varying base constructions. In fact, as [20] pointed out, neither the size nor the packing is a sole indicator of the slip/non-slip regimes. With the base roughness $R_{a}$ defined in Eq. 1, a transition from slip to nonslip is observed. In particular, an intermediate range of $R_{a}$ is found connecting the regime of sliding $\left(R_{a}<0.51\right)$ and nonslip $\left(R_{a}>0.62\right)$. In the intermediate range, either slip or nonslip can occur [20]. Such a phase change is confirmed in Fig. 3, where a non-slip condition is achieved when $R_{a}=0.66$ and 0.72 , regardless of the values of $\Phi$ and $H_{b} / d_{b}$.

\subsection{Bi-disperse flow}

In bi-disperse flows, the effect of base roughness is more complicated due to the occurrence of segregation. Initially, large particles are in contact with the base, giving rise to a relatively larger size ratio $\left(\Phi=d_{l} / d_{b}\right)$ than that after segregation $\left(\Phi=d_{s} / d_{b}\right)$. If we consistently use $d_{l}=2 d_{s}$ and $d_{b}=d_{s}$, the actual size ratio between flowing particles (near the base) and base particles will vary from 2 to 1 during segregation. As a result, the geometric roughness $R_{a}$ becomes a function of time, with which the basal condition evolves. In Fig. 4, the same bi-disperse mixture is flowing on three bases with different constructions, i.e. $H_{b} / d_{b}=1.0,1.6,1.8$ [see Figs. 1(b)-1(d)], and the evolution of base roughness (thus basal velocity) is dependent on the progress of segregation.

To monitor the degree of segregation, we follow [19] to define a state parameter $\alpha=\left(1-\Delta c / \Delta c_{0}\right) / 2$, where $\Delta c=c_{s}-c_{l}$ is the distance between the centers of mass of small $\left(c_{s}\right)$ and large $\left(c_{l}\right)$ particles, and $\Delta c_{0}$ is the initial distance; $\alpha=0$ is the initial state and $\alpha=1$ means perfect segregation. Figure 4(a) presents the temporal development of $\alpha$, which exhibits an exponential growth [15, 19].

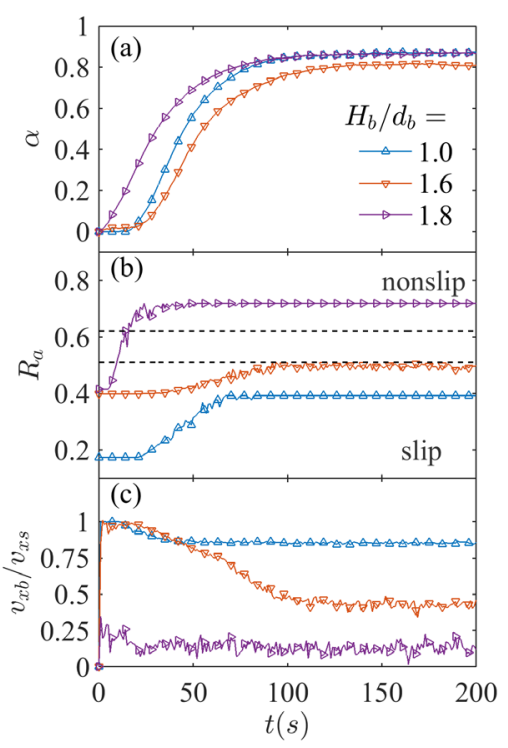

Figure 4. Basal effect on bi-disperse flows $\left(d_{b}=d_{s}, d_{l}=2 d_{s}\right.$, $\theta=25^{\circ}$ ). Evolution of (a) degree of segregation $\alpha$, (b) base roughness $R_{a}$, (c) the normalised base velocity $v_{x b} / v_{x s}$. Two dashed lines in (b) mark $R_{a}=0.51$ and 0.62 , respectively.

The initial base roughness is generally low $\left(R_{a} \leqslant 0.4\right)$ because the base is made of small particles; as segregation occurs and smaller particles reach the base, $R_{a}$ increases until the maximum value is reached [Fig. 4(b)]. The values of $R_{a}$ can be found in three zones: slip, nonslip and the transition in-between. The dashed lines in Fig. 4 mark $R_{a}>0.62$ for the slip zone and $R_{a}<0.51$ for the non-slip zone. Note that the three zones are established in monodisperse flows [20], which still predict well the basal velocity conditions in the current segregation configurations. In Fig. 4(c), we plot the evolution of the normalised base velocity, $v_{x b} / v_{x s}$, where $v_{x b}$ and $v_{x s}$ are velocities at the base and surface layers, respectively. It can be observed that when $H_{b} / d_{b}=1.0$, the varying $R_{a}$ remains in the slip zone, and the base velocity of the flow is high $\left(v_{x b} / v_{x s}=1\right.$, indicating no shear); when $H_{b} / d_{b}=1.6, R_{a}$ evolves from slip zone to the transition zone, which corresponds to the drop of $v_{x b} / v_{x s}$ from 1 to $\sim 0.4$; when $H_{b} / d_{b}=1.8$, nonslip condition is respected for the whole duration (except the initial short time) and $v_{x b} / v_{x s}$ remains small. Another interesting observation is that as basal sliding takes place, the process of segregation is delayed (see $t<20 \mathrm{~s}$ in Fig. 4). It is reported in $[19,21]$ that the sliding near the base may result in crystallisation in the lower layers of the flow, which hinders the development of shear and hence the observed delay of segregation. As small particles percolate towards the base, the crystallised structure can not sustain and then the degree of segregation grows rapidly.

After segregation, a steady state is reached in all presented bi-disperse flows. As shown in Fig. 5(a), the velocity profile for $H_{b} / d_{b}=1.8$ obeys Bagnold's scaling $[1,11,12,21]$, with no sliding at the base. For $H_{b} / d_{b}=1.0$ and 1.6, basal sliding arises and leads to a shift of the velocity profiles. Figure $5(\mathrm{~b})$ shows the profiles of shear rate 

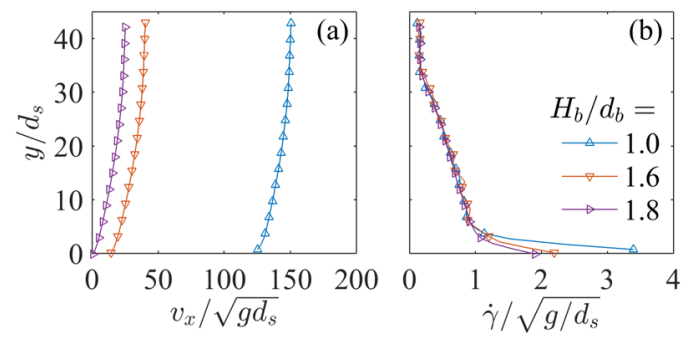

Figure 5. Basal effect on the steady state of bi-disperse flows $\left(d_{b}=d_{s}, d_{l}=2 d_{s}, \theta=25^{\circ}\right)$. (a) Velocity profile. (b) Shear rate profile.

$(\gamma)$ for all three cases; the velocity gradients are identical in the bulk of the flows. This indicates that although basal sliding may delay segregation and significantly change the overall speed of the flow, the development of shear is marginally affected provided that a steady state is reached. Note that if basal sliding happens in a perfectly monodisperse flow, it is possible that crystallisation sustains in the flow, which might lead to an unsteady or plug-like flow where shear is less efficient [5, 20, 21].

\section{Conclusion}

This work presents the effect of geometric base roughness in granular chute flows. A roughness indicator $R_{a}$ is used to represent a variety of roughness values due to different sizes and distributions of base particles. A phase transition from slip $\left(R_{a}<0.51\right)$ to nonslip $\left(R_{a}>0.62\right)$ regimes [20] is validated for both mono- and bi-disperse flows. Basal sliding occurs at low values of $R_{a}$, which mainly shifts the flow velocity to a higher magnitude but has a marginal influence on the shape of velocity profiles. In bi-disperse flows, the basal condition is a function of time because segregation takes place and $R_{a}$ evolves with the progress of segregation. When large particles contact the base at the initial stage, significant sliding may arise and the development of segregation can be delayed. As small particles percolate towards the bottom, non-slip condition can be respected if $R_{a}$ increases beyond 0.62 .

In the future, we expect the roughness indicator $R_{a}$ to be extended to more configurations, such as rotating drum, circular shear apparatus, and curved incline. It would facilitate the characterisation of geometric roughness and the interpretation of the boundary effect on mixing, segregation, and other granular pattern problems.

\section{Acknowledgements}

The work was supported by Research Grants Council of Hong Kong (Grant No. RGC/GRF 17203614), and the Research Institute for Sustainable Urban Development at The Hong Kong Polytechnic University, and FAP-DF, Brazil. This research is conducted in part using the research computing facilities and advisory services offered, by Information Technology Services, the University of Hong Kong.

\section{References}

[1] O. Pouliquen, Physics of Fluids 11, 542 (1999)

[2] O. Pouliquen, J. Delour, S.B. Savage, Nature 386, 816 (1997)

[3] M.J. Woodhouse, A.R. Thornton, C.G. Johnson, B.P. Kokelaar, J.M.N.T. Gray, Journal of Fluid Mechanics 709, 543 (2012)

[4] J.L. Baker, C.G. Johnson, J.M.N.T. Gray, Journal of Fluid Mechanics 809, 168 (2016)

[5] L.E. Silbert, G.S. Grest, S.J. Plimpton, D. Levine, Physics of Fluids 14, 2637 (2002)

[6] V. Kumaran, S. Maheshwari, Physics of Fluids 24, 053302 (2012)

[7] V. Kumaran, S. Bharathraj, Physics of Fluids 25, 070604 (2013)

[8] T. de Haas, L. Braat, J.R.F.W. Leuven, I.R. Lokhorst, M.G. Kleinhans, Journal of Geophysical Research: Earth Surface 120, 1949 (2015)

[9] R. Delannay, M. Louge, P. Richard, N. Taberlet, A. Valance, Nature Materials 6, 99 (2007)

[10] W. Zhou, Z. Lai, G. Ma, L. Yang, Y. Chen, Granular Matter 18 (2016)

[11] L.E. Silbert, D. Ertaş, G.S. Grest, T.C. Halsey, D. Levine, S.J. Plimpton, Physical Review E 64, 051302 (2001)

[12] A. Tripathi, D.V. Khakhar, Physics of Fluids 23, 113302 (2011)

[13] T. Weinhart, A.R. Thornton, S. Luding, O. Bokhove, Granular Matter 14, 531 (2012)

[14] A.R. Thornton, T. Weinhart, S. Luding, O. Bokhove, The European Physical Journal E 35 (2012)

[15] L. Staron, J.C. Phillips, Physics of Fluids 26, 033302 (2014)

[16] D.R. Tunuguntla, O. Bokhove, A.R. Thornton, Journal of Fluid Mechanics 749, 99 (2014)

[17] D.R. Tunuguntla, A.R. Thornton, T. Weinhart, Computational Particle Mechanics 3, 349 (2016)

[18] U. D'Ortona, N. Thomas, R.M. Lueptow, Physical Review E 93, 022906 (2016)

[19] L. Jing, C.Y. Kwok, Y.F. Leung, Discrete element modelling of grain size segregation in bidisperse granular flows down chute, in International Conference on Particle-Based Methods, Fundamentals and Applications (Barcelona, Spain, 2015), http://hub.hku.hk/handle/10722/213642

[20] L. Jing, C.Y. Kwok, Y.F. Leung, Y.D. Sobral, Physical Review E 94, 052901 (2016)

[21] L. Jing, C.Y. Kwok, Y.F. Leung, Y.D. Sobral, Basal Effect in Mono- and Bi-Disperse Chute Flows, in 7th International Conference on Discrete Element Methods (Dalian, China, 2016)

[22] L. Staron, J.C. Phillips, Physical Review E 92, $022210(2015)$

[23] MATLAB Release 2016b, The MathWorks, Inc., Natick, Massachusetts, United States (2016) 